\title{
Equidad de género en docentes líderes de la Universidad Central del Ecuador, en el contexto del Objetivo de Desarrollo Sostenible 5
}

Gender Equity in Leading Teachers at the Central University of Ecuador, in the Context of Sustainable Development Goal 5

Equidade de gênero em docentes líderes da Universidad Central del Ecuador no contexto do Objetivo de Desenvolvimento Sustentável 5 (Objetivo de Desarrollo Sostenible 5)

\section{Sandra Almeida-Guzmán \\ Universidad de Huelva. Huelva, España salmeidaguz@gmail.com https://orcid.org/0000-0003-3391-8574}

\section{María de la O Barroso-González \\ Universidad de Huelva. Huelva, España mariadelaog@gmail.com \\ https://orcid.org/0000-0002-1507-4745}

DOI: https://doi.org/10.32719/25506641.2020.8.4

Recibido: 6 de febrero de 2020 - Revisado: 3 de abril de 2020

Aceptado: 16 de mayo de 2020

$$
\begin{aligned}
& \text { Artículo de investigación } \\
& \text { Licencia Creative Commons }
\end{aligned}
$$




\section{Resumen}

La presente investigación analiza las condiciones de equidad de género en las que se encuentran las docentes que ocupan posiciones de liderazgo en la Universidad Central del Ecuador (UCE) desde la perspectiva del Objetivo de Desarrollo Sostenible (ODS) 5 y su meta 5.5, para lo cual se utilizó una metodología mixta de carácter cuantitativo y cualitativo.

Si bien la problemática de género en las universidades está identificada y presenta una serie de implicaciones y/o factores como la cultura androcéntrica, la falta de referentes históricos, la feminización de los espacios privados, los ámbitos organizativos mínimos para mujeres al interior de las universidades, la falta de conciencia de género, las diferencias de estilos de liderazgo (prototipos), la universidad como espacio reproductor de desigualdades, etc., ante lo cual se han establecido acuerdos, políticas y metas, aunque en la práctica los avances son lentos.

En el caso ecuatoriano, el marco jurídico ha ejercido un rol fundamental, ya que ha presionado a las universidades a incorporar mujeres en puestos de liderazgo y dirección. Los hallazgos evidencian que el cumplimiento del ODS 5 y su meta 5.5 en la Central presenta avances, pero no suficientes.

Palabras clave: Objetivos de Desarrollo Sostenible, equidad de género, docencia universitaria, liderazgo.

JEL: D63 Equidad, justicia, desigualdad y otros criterios normativos y de medida.

\section{Summary}

The present investigation analyses the conditions of gender equality in which the teachers who occupy leadership positions at the Central University of Ecuador (UCE) are found from the perspective of the Sustainable Development Goal (SDG) 5 and its goal 5.5, for which a mixed quantitative and qualitative methodology was used.

Although the gender problem in universities is identified and presents a series of implications and/or factors such as the androcentric culture, the lack of historical references, the feminization of private spaces, the minimum organizational areas for women within the universities, lack of gender awareness, differences in leadership styles (prototypes), the university as a reproductive space for inequalities, etc., in response to which agreements, policies, goals, and more have been established; in practice progress is slow.

In the Ecuadorian case, the legal framework has played a fundamental role since it has pressured universities to incorporate women into leadership and management positions. The findings show that compliance with SDG 5 and goal 5.5 at the Central University shows progress, but not enough.

Keywords: Sustainable Development Goals, gender equity, university teaching, leadership.

JEL: D63 Equity, Justice, Inequality and other normative criteria and measures. 


\section{Resumo}

A presente pesquisa analisa as condições de equidade de gênero nas quais se encontram as docentes que ocupam posições de liderança na Universidad Central del Equador (UCE) a partir da perspectiva do Objetivo de Desenvolvimento Sustentável (ODS) 5 e sua meta 5.5. Para tanto, utilizou-se uma metodologia mista de caráter quantitativo e qualitativo.

Embora a problemática de gênero nas universidades esteja identificada e apresente uma série de implicações e/ou fatores, como a cultura androcêntrica, a falta de referentes históricos, a feminização dos espaços privados, os âmbitos organizativos mínimos para as mulheres no interior das universidades, a falta de consciência de gênero, as diferenças de estilos de liderança (protótipos), a universidade como espaço reprodutor de desigualdades, etc., ante os quais foram estabelecidos acordos, políticas, metas, etc., na prática, os avanços são lentos.

No caso equatoriano, o marco jurídico exerceu um papel fundamental ao pressionar as universidades a incorporar mulheres a cargos de liderança e direção. Os resultados evidenciam que o cumprimento do ODS e de sua meta 5.5 na Universidad Central apresenta avanços, porém não suficientes.

Palavras-chave: Objetivos de Desenvolvimento Sustentável, equidade de gênero, docência universitária, liderança.

JEL: D63 Equidade, justiça, desigualdade e outros critérios e medidas normativos.

\section{Introducción}

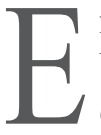

n el marco de los objetivos de desarrollo sostenible de las Naciones Unidas, en adelante ODS, el Objetivo 5 persigue "lograr la igualdad entre los géneros y empoderar a todas las mujeres y niñas", buscando a través de su meta 5.5: "Asegurar la participación plena y efectiva de las mujeres y la igualdad de oportunidades de liderazgo a todos los niveles decisorios en la vida política, económica y pública". La presente investigación analiza las condiciones de equidad de género de las docentes que ocupan posiciones de liderazgo en la Universidad Central del Ecuador (UCE), la segunda universidad más grande del país.

A partir de 1975, cuando las Naciones Unidas declararon el año Internacional de la Mujer, se empieza a tomar conciencia sobre la importancia de impulsar la equidad de género a través de mayor acceso a educación y trabajo femenino. Las universidades, desde el siglo XX, han tenido un proceso significativo de feminización entre las estudiantes, tanto en sus tasas de acceso 
como de egreso. En consecuencia, también se ha incrementado el porcentaje de mujeres profesionales dedicadas a la docencia universitaria; sin embargo, su nivel de representatividad aún es bajo. Por ejemplo, en España, el informe Cientificas en Cifras (ES Ministerio de Ciencia, Innovación y Universidades 2017) señala que solo el $27 \%$ de decanatos están en manos de mujeres y el porcentaje es significativamente menor cuando se trata de rectorados: $2 \%$ de mujeres ocupa este cargo.

Según el artículo publicado por la universidad en línea UNIR (2020), seis de cada diez estudiantes universitarios en Ecuador son mujeres; además, menciona que hace apenas tres décadas eran solo el $40 \%$ del total. Este proceso de mayor participación femenina en las universidades no se presenta en la docencia, que ha incrementado su número, pero no en forma proporcional a las estudiantes, y es aún menor en cargos de dirección. Para entender las razones es necesario realizar un análisis tomando en cuenta una serie de implicaciones y/o factores como la cultura androcéntrica, la falta de referentes históricos, la feminización de los espacios privados, los ámbitos organizativos mínimos para mujeres al interior de las universidades, la falta de conciencia de género, las diferencias en los estilos de liderazgo (prototipos), la universidad como espacio reproductor de desigualdades, entre otros.

Para evidenciar lo mencionado se ha tomado como referente a la UCE, en la cual se puede observar un paulatino incremento de mujeres docentes, así como también una mayor participación en puestos de decisión, sin alcanzar la equidad. Existe una baja intervención femenina en temas de investigación y publicaciones, por tanto, se considera importante conocer las causas y verificar el cumplimiento de la meta de paridad de género de los ODS.

En este contexto, el objetivo de la presente investigación es analizar las condiciones de equidad de género en las que se encuentran las docentes que ocupan posiciones de liderazgo en la UCE. Se planteó como pregunta de investigación; ¿se está dando cumplimiento al ODS 5 y su meta 5.5 en el caso de las mujeres docentes en posición de liderazgo, respecto a puestos de elección, de designación y aportes en investigación en la UCE? Para cumplir con este objetivo se realizó un trabajo de tipo cualitativo basado en un focus group con docentes universitarias y una revisión de las estadísticas de la UCE. 
Los resultados obtenidos se presentan en cuatro apartados: una revisión de la principal literatura sobre equidad de género y sus vínculos con la educación universitaria; descripción de la metodología; los resultados obtenidos en la aplicación del objetivo 5 de los ODS y las conclusiones.

\section{Revisión de la literatura}

\section{Equidad de género y docencia universitaria}

La equidad de género, en docentes universitarias, se encuentra atravesada por una serie de factores, tanto visibles como invisibles, que limitan el desarrollo de las mujeres, especialmente en ámbitos en los cuales su nivel de participación es bajo, como la investigación y la dirección de la universidad. Existe un marcado dominio de los hombres, que resulta contraproducente en un espacio considerado generador de conocimiento, compromiso con la sociedad, igualitario y no reproductor de las desigualdades.

Como se mencionó anteriormente, entre los factores visibles se puede mencionar la estructura patriarcal de la sociedad y la predominancia del ámbito privado (tradicionalmente la mujer es la responsable del hogar) por sobre el público. Los factores invisibles tienen que ver con formas simbólicas que influyen en la pérdida de confianza, puesto que el sistema sexo-género hace que la sociedad vea a la mujer por fuera del campo de lo racional y con menos capacidad para asumir espacios de decisión, que conlleva a bajos niveles de autoestima de las mujeres y su autoexclusión. En este sentido, según el artículo "Las voces de las líderes de la Universidad de Alicante sobre cuestiones de género", de Martínez y Urrea (2018, 22), las limitaciones se encuentran dentro de las mismas académicas, que no reconocen la influencia del género en sus dificultades profesionales y no logran identificar los comportamientos y actitudes discriminatorios.

Otro de los retos cualitativos-simbólicos que afectan la equidad de género en las docentes es la percepción de la existencia de áreas disciplinarias menos reconocidas a las que se otorga menor valor científico, y es precisamente en estas áreas donde existe mayor concentración femenina, como en salud y cuidados (medicina, enfermería), y en educación. En Ecuador se con- 
firma esta apreciación, de acuerdo con el artículo de El Comercio (2018), con los siguientes datos: participación femenina como investigadoras acreditadas en educación, $52 \%$; medicina, $48 \%$; ciencias sociales, $50 \%$; en tanto que en ciencias físicas y matemáticas, $26 \%$ y en ingeniería $25 \%$.

En el proceso de consecución de la equidad de género en posiciones de liderazgo, también se aborda sobre los estilos de su ejercicio, según Merma y Ávalos $(2018,153)$ :

Las mujeres están adoptando posiciones de liderazgo, pero no de forma igualitaria que los hombres, ni cuantitativamente ni cualitativamente. Mientras las mujeres destacan la confianza de sus compañeros, el saber desenvolverse y el tener valores personales y consideración individualizada, rasgos tradicionalmente atribuidos al liderazgo transformacional, en los hombres prevalecen cualidades como: mejorar la enseñanza-aprendizaje, servir de puente entre los profesores y otros grupos, y tener capacidad de gestionar conflictos, en la perspectiva del liderazgo transaccional.

Las mujeres muestran una tendencia más comunal, definida por una mayor preocupación por los demás, la sensibilidad, amabilidad y cordialidad. Este tipo de liderazgo desarrolla los niveles de conciencia y compromiso con las organizaciones.

La brecha de género en la academia es más evidente en actividades investigativas; la mayoría de mujeres considera que su condición les afecta negativamente, a lo que se suman los inconvenientes que se presentan por los permisos de maternidad. De acuerdo con un artículo publicado en el diario El Mundo de España (2017), se menciona que a pesar de que las mujeres tienen valoraciones más óptimas en sus puestos que los hombres, sacan mejores notas de media en grados y posgrados, son pocas las que consiguen llegar a lo más alto del escalafón de la docencia, y la relación con la media de catedráticas en las universidades públicas españolas es solo del $21 \%$. Esther Escolano (2006), subdirectora general de Estudios y Cooperación de la Universidad de Valencia, menciona que cuando una mujer intenta llegar a puestos de responsabilidad, sea de cátedra o posición directiva, se tienen en cuenta factores distintos al mérito y capacidad; en tal sentido, menciona que entra en juego el saber moverse, los grupos y el lobbying, hay que estar todo el tiempo trabajando en eso y que allí las mujeres se retiran un poco. 
En cuanto a la participación de las docentes en investigación, de acuerdo con el mismo artículo del diario El Mundo (2017), las mujeres suelen ser mayoría entre los investigadores de los centros universitarios públicos, pero solo hasta cierto momento: los 34 años, edad en la que hombres y mujeres concluyen su doctorado, requerimiento para la cátedra. A partir de los 35 años, la situación se invierte: los hombres son los que mayoritariamente se dedican a la investigación. Esta situación se explica porque las mujeres optan por la maternidad.

En Ecuador, la participación femenina en la docencia universitaria tiene indicadores más interesantes que los españoles, según el artículo de $E l$ Comercio: "Una mayor participación femenina todavía es un reto en el país". Para 2013, el 36\% de docentes son mujeres, frente a un $64 \%$ de hombres; y a nivel de rectores, un $18 \%$ de participación femenina.

\section{Los ODS de las Naciones Unidas: alcances y limitaciones}

En 2015, en la Asamblea de las Naciones Unidas, 193 países adoptaron la Agenda 2030 para el Desarrollo Sostenible, que fue producto de un extenso proceso de negociaciones y acuerdos previos e implementada a partir de

Figura 1

\section{Los 17 objetivos de desarrollo sostenible}
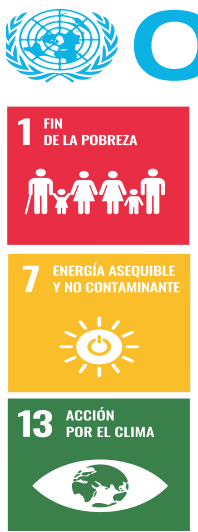
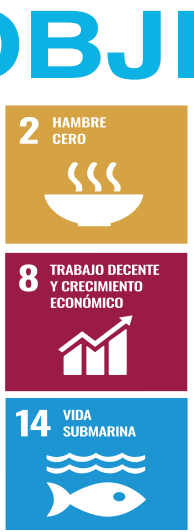
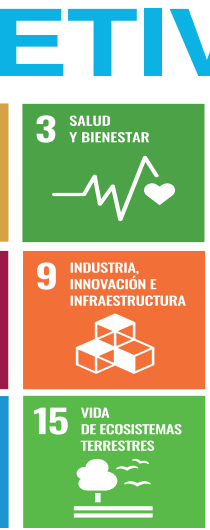
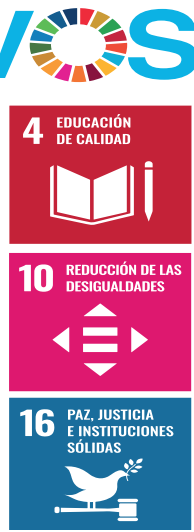
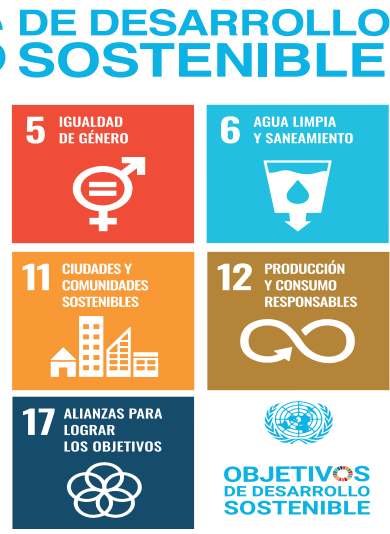
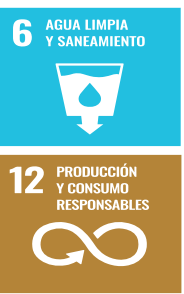

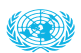

OBJETIVOS SOSTENIBLE

Fuente: Naciones Unidas (2015). 
enero de 2016; se espera cumplir con 17 objetivos de desarrollo sostenible. Según Gómez $(2017,107)$ : "los ODS contienen la agenda global más ambiciosa aprobada por la comunidad internacional para movilizar la acción colectiva en torno a objetivos comunes". En la figura 1 se pueden apreciar en detalle los ODS.

Los 17 ODS cuentan con 169 metas asociadas y 230 indicadores para medir su cumplimiento. Los ODS se encuentran estrechamente vinculados y tienen que ver con la innovación, protección al medioambiente y equidad, y cubren todas las dimensiones de acción del ser humano: social, económico, político y ambiental.

Entre las principales críticas a la propuesta de la Agenda 2030 se pueden mencionar las siguientes:

- Se presentan varias contradicciones entre los países firmantes como: el Objetivo 16 compromete a todos los Estados a "promover sociedades pacíficas", cuando los países occidentales signatarios de los acuerdos son los principales vendedores de armas del mundo; o el Objetivo 13, que obliga a "adoptar medidas urgentes para combatir el cambio climático", mientras hay países que niegan incluso que este fenómeno exista (Fundación FUNEM 2019).

- Los ODS se consideran en gran medida idealistas; no se dispone de indicadores en los países más pobres, existen metas de difícil cuantificación, y se presentan ciertas diferencias entre las estadísticas nacionales e internacionales, que no permite una adecuada evaluación de avance de las metas planteadas.

- Los ODS incorporan elementos novedosos en su diseño e integran las tres dimensiones de desarrollo sostenible: económica, social y ambiental, priorizando la lucha contra la pobreza y el hambre, pero con un fuerte anclaje en la defensa de los derechos humanos, la igualdad de género y el empoderamiento de las mujeres, abordando la reducción de las desigualdades dentro de cada país y en los diferentes Estados como elemento prevalente, junto con la eliminación de patrones de consumo insostenibles (Naciones Unidas 2016).

- Presentan una visión del crecimiento económico incluyente y sostenible, respetuoso con la salud del planeta y de la población. 
- "Es evidente que los ODS, como producto del sistema internacional de Naciones Unidas, no cuestionan elementos fundamentales de las sociedades occidentales, como son la modernidad, el capitalismo y el antropocentrismo. Por lo tanto, el resultado de su posible consecución no puede contribuir a la mejora del bienestar de la humanidad, o al menos de aquella parte de la humanidad que cuestiona dichos elementos; esto es los decolonialistas, los poscapitalistas y los biocentristas" (Hidalgo et al. 2018, 18).

- "Las desigualdades de género que hay que combatir son las referentes a las oportunidades y al reparto del poder; pues los roles sociales que cada género desempeñe en una sociedad no tienen por qué ser iguales, ya que dichos roles dependen del papel que las diferentes culturas juegan en la sociedad" (Hidalgo et al. 2018, 30).

En los ODS, el Objetivo 5 posee un efecto catalizador para la consecución de la Agenda 2030; el cambio transformador se puede dar a través de políticas de género. Pues queda claro que:

Si se facilita a las mujeres y niñas igualdad en el acceso a la educación, atención médica, un trabajo decente y representación en los procesos de adopción de decisiones políticas y económicas, se impulsarán las economías sostenibles y se beneficiará a las sociedades y a la humanidad en su conjunto. (ONU 2019)

En síntesis, las nueve metas propuestas por el ODS 5 (ONU 2015) son las siguientes:

1. Fin de la discriminación contra mujeres y niñas.

2. No violencia, en ámbitos públicos y privados.

3. Sin prácticas nocivas como matrimonio infantil, mutilación femenina, etc.

4. Valorar trabajo no remunerado (cuidados y trabajo doméstico).

5. Participación e igualdad de oportunidades de liderazgo en todos los niveles políticos, económicos y públicos.

6. Acceso a salud sexual y reproductiva.

7. Derecho a recursos económicos en igualdad de condiciones, propiedades y otros bienes.

8. Acceso a TIC para promover el empoderamiento de la mujer. 
9. Desarrollo de políticas en busca de la igualdad de género y empoderamiento de mujeres y niñas a todos los niveles.

La principal crítica al ODS 5 es el no incluir a grupos alternativos como LGBTI (lesbianas, gais, bisexuales y transgénero), así como el cálculo de indicadores, pues muchos países no cuentan con información estadística necesaria. Las nueve metas mencionadas cuentan con 14 indicadores que permiten valorar su avance. De las metas propuestas en el Objetivo 5, la presente investigación centra su atención en la 5.5, el indicador a utilizar es el 5.5.2 (proporción de mujeres en cargos directivos).

\section{Marco jurídico de la docencia universitaria en Ecuador}

Según el artículo 26 de la Constitución de la República del Ecuador, el Plan Nacional Toda una Vida, la Agenda Nacional de las Mujeres y la Igualdad de Género, la Ley Orgánica de Educación Superior (LOES) y su reforma de 2018 y el Reglamento de Régimen Académico presentan avances a favor de la equidad de género. Determinan que la autonomía responsable, de la cual gozan las universidades, debe incorporar el principio de equidad de género para estructurar y conformar sus órganos de gobierno. El artículo 56 de la LOES establece que las universidades deben incorporar los principios de paridad de género, igualdad de oportunidades y equidad:

La elección de rector o rectora, vicerrectores o vicerrectoras, y de los representantes de los distintos estamentos ante los órganos de cogobierno en las instituciones de educación superior, se realizará a través de listas que deberán ser integradas respetando la alternancia, la paridad de género, igualdad de oportunidades y equidad conforme a la Constitución. (EC 2018, art. 56)

El cumplimiento de este mandato ha permitido, por ejemplo, que una de las universidades más importante de la ciudad de Quito, como la Escuela Politécnica Nacional, por primera vez en su historia esté liderada por una mujer.

Por tanto, la LOES presenta interesantes avances en equidad de género, pero también existen cuestionamientos de las docentes a sus altas exigencias, pues su legislación mantiene fuera de puestos de liderazgo a la gran mayoría de mujeres que, por ejemplo, para acceder al cargo de rector debe cumplir requisitos como: debe ser ocupado por un docente regular y a tiempo com- 
pleto, con $\mathrm{PhD}$, experiencia de por lo menos 5 años en gestión educativa universitaria, publicaciones de por lo menos 6 obras y/o artículos indexados, haber accedido a la docencia a través de concurso público, experiencia como docente y/o investigación de por lo menos 5 años, además de ejercer la docencia con probidad, eficiencia y pertinencia. Existe, por tanto, la dificultad de las mujeres en dedicarse al cien por ciento de tiempo a la vida pública, puesto que también es responsable de su ámbito privado.

Los organismos rectores del sistema de educación superior son el Consejo de Educación Superior (CES), a cargo de la planificación, regulación y coordinación interna; el Consejo de Evaluación, Acreditación y Aseguramiento de la Calidad de la Educación Superior (CEAAES), actualmente Consejo de Aseguramiento de la Calidad de la Educación Superior (CACES), encargado del aseguramiento de calidad en la educación superior a través de procesos de evaluación, acreditación y categorización de las instituciones de educación superior; y la Secretaria de Educación Superior, Ciencia Tecnología e Innovación (SENESCYT), que ejerce la rectoría de la política pública de la educación superior y coordina acciones con el Ejecutivo y las instituciones de educación superior.

Es importante tomar nota de la influencia ejercida por organismos internacionales, que son parte de ONU Mujeres, como el Tratado sobre la Eliminación de Todas las Formas de Discriminación Contra la Mujer (CEDAW), considerado como el principal instrumento jurídico para la promoción de los derechos de las mujeres, obligando a los Estados a reformar leyes para conseguir este fin. Ecuador, al ser suscriptor de este convenio, debe establecer mecanismos en busca de la equidad de género en todos los niveles; para el caso de la educación superior se han establecido los organismos rectores mencionados en el párrafo anterior que, entre otras responsabilidades, tienen la consecución de la equidad. Por ejemplo, el CACES en 2013 incluyó la paridad de género (porcentaje de mujeres respecto al porcentaje de hombres) como indicador de la calidad de la docencia, que es el mismo establecido para verificar el cumplimiento del ODS 5 y su meta 5.5. 


\section{Metodología}

Se utilizó una metodología mixta de carácter cuantitativo y cualitativo. En lo referente a la investigación cuantitativa, como estrategia de investigación, se parte de la revisión bibliográfica, búsqueda extensiva en las bases de datos disponibles en internet y en el archivo de la UCE: número de docentes y autoridades mujeres; su participación en investigaciones y su vinculación con la comunidad. Se parte del abordaje de un número considerable de documentos de relevancia sobre la situación actual de género en la docencia universitaria.

Está metodología ha sido enriquecida con el aporte cualitativo a través de un focus group realizado en la Universidad Andina Simón Bolívar, Sede Ecuador, con las docentes más representativas y expertas en el tema de género, lo que permitió reforzar los procesos de discusión y análisis.

El análisis se vinculó desde la perspectiva del ODS 5 y cumplimiento de su meta 5.5 aplicando el indicador ODS (proporción de mujeres en cargos directivos) para verificar su cumplimiento en la UCE, para finalmente establecer las principales conclusiones y recomendaciones.

\section{Resultados}

\section{Alcances ODS 5 en Ecuador}

El avance del Ecuador en el cumplimiento de los ODS, según el SDG Index \& Dashboard en 2017, se sitúa en la posición 71 de 149 con 60,69 puntos, 2,28 por encima de la media mundial. En cuanto a la situación de los ODS en el Ecuador, el estudio de la AECID (2017) sobre la contribución de la empresa a la Agenda de los ODS se encontró que los tres ODS más avanzados son: acción por el clima (ODS 8), igualdad de género (ODS 5), y trabajo decente y crecimiento económico (ODS 8). En tanto que los tres ODS con menos avances son: industria, innovación e infraestructura (ODS 9), producción y consumos responsables (ODS 12), y hambre cero (ODS 2).

La consecución de ciertos ODS es un tema que puede variar, en la medida que en países menos desarrollados los resultados proporcionados por 
indicadores son más vulnerables a las crisis económicas; por ejemplo, actualmente el trabajo decente y crecimiento económico en Ecuador no es de los más avanzados (ODS 8).

De acuerdo con el Análisis Voluntario Ecuador 2018 (SENPLADES ${ }^{1}$ 2018), para verificar el avance del país en el cumplimiento de la Agenda 2030, para el caso del ODS 5, el gobierno incluyó en el Plan Nacional de Desarrollo 2017-2021 Toda una Vida la erradicación de la violencia de género, tanto para mujeres como para personas de la comunidad LGBTI. Presenta como uno de los avances la reducción de la brecha salarial entre hombres y mujeres, del $23 \%$ registrado en 2014 cae al $20 \%$ en 2017. En lo referente a violencia de género, las estadísticas, para mujeres, señalan que un $60 \%$ han vivido algún tipo de violencia, $40 \%$ ha sufrido violencia sexual y $53,9 \%$ han estado expuestas a violencia psicológica, siendo la última la más frecuente (INEC 2011).

\section{Focus group "Situación de género en la docencia universitaria"}

Para el análisis de la equidad de género en la UCE, la investigación tenía prevista la realización de entrevistas semiestructuradas a mujeres que se encuentran en puestos de decisión, pero dados los requerimientos internos de la UCE, ${ }^{2}$ se decidió realizar una investigación de tipo exploratoria, recurriendo a varias y recientes publicaciones realizadas en la UCE que abordan esta temática.

Se considera un aporte relevante, de tipo cualitativo, el focus group realizado en la Universidad Andina Simón Bolívar, Sede Ecuador, el 14 de septiembre de 2019, que contó con la presencia de importantes representantes de género en Ecuador y España.

Entre los principales resultados generales se mencionan los siguientes:

- Es importante hablar de género desde las universidades, ya que constituyen un actor territorial mayúsculo para poner en marcha muchos pro-

1. Actualmente Secretaría Técnica Planifica Ecuador.

2. Un requerimiento de la UCE para investigaciones es que estas deben ser aprobadas por el Comité de Ética y sus comisiones, lo cual resultaba un proceso largo, especialmente para una investigadora externa. 
cesos y su responsabilidad social es elevada. Se debe trabajar el tema de género de manera transversal, en el pénsum académico, en tareas, investigaciones y otros, lo cual permitirá su empoderamiento.

- Es fundamental que todos (hombres, mujeres y otras identidades sexo-genéricas) se impliquen en el tema, ya que superar la inequidad de género no solo compete a mujeres, sino a todos los seres humanos.

- Se debe realizar un análisis situacional con énfasis en los puntos críticos entre el diseño de políticas y normas, así como resultados de su implementación en las universidades.

- No se debe analizar la equidad de género desde la mirada funcionalista, como brechas, pues no es suficiente, más bien es importante construir indicadores más complejos; algunas veces las estadísticas no reflejan la realidad de la situación, por lo cual es necesario profundizar más y desarrollar indicadores, tanto cuantitativos como cualitativos, que evidencien la calidad, pertinencia y utilidad de las investigaciones.

- El análisis debe destacar los desarrollos de las docentes universitarias en sus tres ámbitos de acción: docencia, investigación y vinculación con la comunidad, en los que existe una serie de factores a considerar, como el tipo de vínculo laboral que tienen con la institución. No es lo mismo ser docente principal que ocasional, si hay brecha o no y sus afectaciones, desde qué edad se encuentra como docente y la relación con la edad de los docentes hombres, lo cual permitirá analizar las relaciones entre las oportunidades de hombres y mujeres.

- En la vinculación con la sociedad, se debe ver qué aportes están haciendo y desde qué campos, tomar en cuenta otras formas de vinculación, como asesoramiento a organizaciones de mujeres, apoyo jurídico y otros; actividades que tienen dimensión académica si se mira a la universidad desde abajo.

- En el ámbito de investigación es donde se encuentra una mayor crítica por parte de las docentes: consideran que la LOES promueve la realización de publicaciones indexadas y existe un grupo de mujeres investigadoras que lo hacen desde la resistencia, consideran que lo crítico no es aceptado y no es publicado, están en contra de la lógica del productivismo, que las ven como meras máquinas, y piensan que la cantidad no es lo importante. 
- Existe consenso en las docentes en la inclusión de temas de género en las diferentes líneas de investigación, pues se considera fundamental realizar una evaluación constante sobre sus avances y limitaciones.

- Otro elemento que afecta la equidad de género son las evaluaciones anuales de trabajo, que para el caso de mujeres que estén en permiso de maternidad y/o lactancia, obviamente el reporte será que trabajaron menos, con las visibles consecuencias de una evaluación desventajosa.

- Se debe realizar el análisis de género a nivel de mallas curriculares. Existe la idea del poder que se distribuye entre las asignaturas, porque en la construcción de las mallas hay asignaturas con más poder que otras, hay optativas menos necesarias, por tanto, con menor influencia. Existen estudios que mencionan que habitualmente las asignaturas con más poder la dirigen los hombres, y las otras las mujeres. O si la asignatura a cargo tiene que ver con el estereotipo o rol de género, es decir, tiene relación con el tema educativo, de cuidado, lo cual todavía está inserto en el imaginario. Las cargas horarias también constituyen un estímulo para continuar con la docencia o salir, puesto que pueden programarles horarios que les complica su vida familiar.

- Es indiscutible la existencia en la docencia de relaciones de poder, que genera objeciones, opresiones o espacios de privilegios, formas de violencia, acoso, situación que se presenta con un jefe y también entre pares.

- Algunas docentes han sentido discriminación, porque han sido sutilmente utilizadas por la institucionalidad para demostrar enfoque de género en la universidad, en tanto que en la práctica se encuentran con limitaciones que no les permite superar el esquema patriarcal.

- Es importante construir formas propias de liderazgo, reivindicar características personales de las mujeres como "sensible", "comprometida" y "solidaria", que generan otro tipo de práctica.

- La investigación debe realizarse a través de un enfoque de interseccionalidad, que toma en cuenta clase social, interculturalidad, orientación sexual y discapacidad, las cuales son construidas y están interrelacionadas, y deben ser consideradas mucho más desde la academia, en sus diferentes áreas de acción: docencia, investigación y actividades de vinculación con la comunidad. 
En cuanto a los principales resultados específicos de la situación de género en la UCE se mencionan lo siguiente:

- La UCE no cuenta con políticas sobre equidad de género. Lo que se ha hecho en este sentido no es por política universitaria, sino por la reforma de la LOES (EC 2018), en concordancia con el principio de igualdad y paridad de la Constitución de la República.

- En 2017 el CES emitió una resolución que obliga a las universidades a crear un plan de igualdad. Actualmente se está trabajando en la línea base para hacer una política pública de igualdad dentro de la UCE. Además, el art. 35 de la Ley de Prevención de Violencia contra las Mujeres (EC 2018) establece obligaciones a las instituciones rectoras de educación superior, especialmente a las administradoras CES, SENESCYT y CACES.

- En conclusión, en la UCE no hay políticas que propendan a la equidad de género; por ejemplo, no se cuenta con políticas sobre maternidad de profesoras, bienestar estudiantil, en muchos casos las políticas se han construido desde la omisión.

A pesar de las sugerencias de las expertas en género participantes en el focus group, respecto a la relevancia del ODS 5 en la consecución de la equidad de género, se resolvió continuar con la propuesta inicial y realizar el análisis de la equidad de género en la UCE en el contexto del ODS 5 y su meta, por considerar que es un principio básico de derechos humanos.

El principal argumento es que a pesar de ser lo mínimo exigible, no se ha cumplido en ningún país del mundo. Así lo asevera la portavoz de la Organización para la Cooperación y el Desarrollo Económico (OCDE), Sherpa Gabriela Ramos: "Ningún país del mundo ha alcanzado la igualdad de género. Incluso los más igualitarios ofrecen menos oportunidades para ellas" ( $E l$ Mundo 2017).

\section{Equidad de género en la UCE}

El análisis de la equidad de género en la UCE partirá de la revisión bibliográfica existente. Al respecto, se han identificado varias investigaciones realizadas en los últimos años, tales como: Plan de igualdad de oportunidades en la UCE; género, poder y liderazgo en la UCE; relaciones de género en la UCE, en las cuales se puede apreciar importantes diagnósticos, inclu- 
so planes, políticas y acciones estratégicas que propenden a la equidad de género; pero en la práctica constituyen solo propuestas no implementadas, frente a lo cual se presenta la siguiente inquietud: ¿cuál es el problema en su implementación? La respuesta puede ser falta de decisión política, insuficiente disponibilidad de recursos o poca presión de los grupos interesados.

La UCE también ha tenido un incremento de mujeres a nivel estudiantil; por ejemplo, en 2018 el reporte de matriculados registra que el 57\% corresponde a mujeres y el $43 \%$ a hombres, con una mayor concentración del ingreso femenino en carreras como ciencias médicas; a nivel de docentes, el $34 \%$ es mujer y el $66 \%$ hombre; en cuanto a la participación de las mujeres en el gobierno universitario, un $34 \%$ corresponde a mujeres y $66 \%$ a hombres (Reportes UCE 2018).

En el análisis de la participación femenina en los diferentes ámbitos de acción de la docencia universitaria tenemos:

\section{Docencia}

Hasta el 2018 la UCE no contaba con vicerrectoras, a la fecha no ha tenido una rectora y la participación femenina en Consejo Universitario es baja (34\%), lo cual se explica además por la existencia de una planta docente mayoritariamente conformada por hombres. Para el período 2015 a 2019, la participación femenina presenta una variación significativa respecto a 2013, pasando del 20 al $35 \%$. El período de mayor crecimiento (5\%) corresponde a 2016-2017; en los siguientes años, el crecimiento no supera el 1\%. La docencia masculina en la UCE representa aproximadamente las tres cuartas partes de la femenina, lo que confirma el ejercicio del poder masculino en este campo universitario.

Una visualización sobre la evolución de la participación de la docencia femenina desde 2015 (5 años) se presenta en la siguiente figura: 
Figura 2

Docentes de la UCE

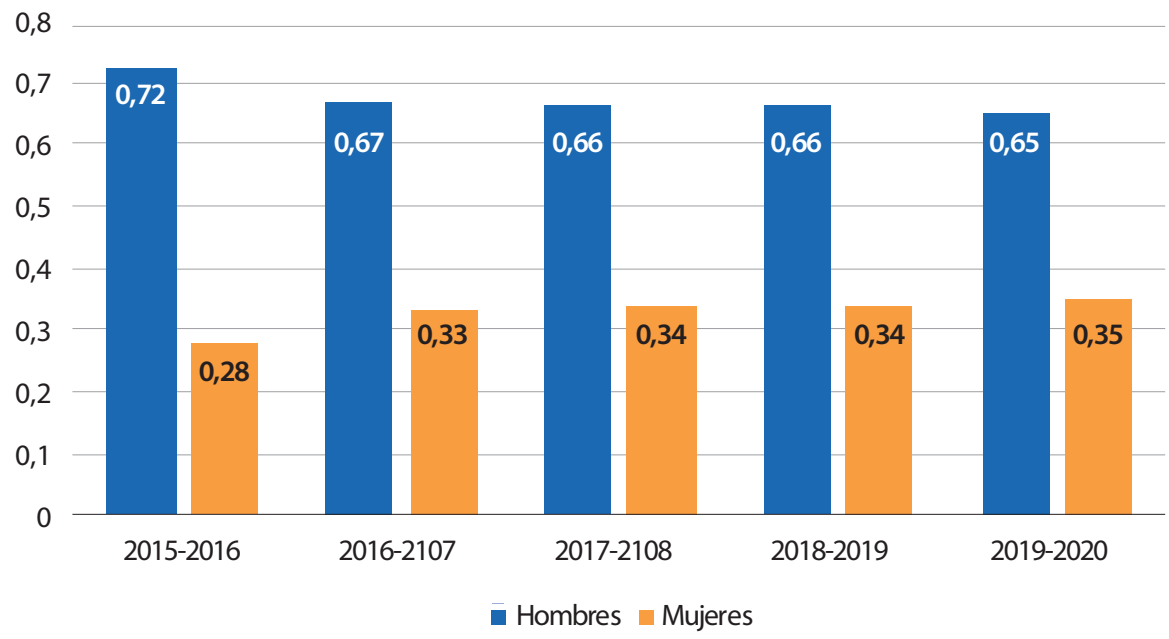

Fuente: UCE (2019).

Elaboración propia.

Se puede apreciar la mayoritaria participación masculina entre facultades, excepto en la de Ciencias Psicológicas. Las facultades con un predominio marcado de hombres son: Administración, Economía, Medicina, Filosofía, Ingeniería y Jurisprudencia (ver figura 3).

\section{Actividades de investigación}

La participación femenina en este ámbito era marginal. En las convocatorias 2012-2013, de los 19 proyectos aprobados, solo 4 (21\%) fueron dirigidos por mujeres. En 2012, de los 48 docentes con $\mathrm{PhD}$, únicamente 8 $(16,6 \%)$ son mujeres. En publicaciones de libros en 2013, solo se registra la participación de 16 hombres y no hay publicaciones de mujeres. Para 2018, se presenta un avance: de los 83 proyectos aprobados, 24 son dirigidos por docentes mujeres $(29 \%)$, en tanto que 59 por hombres $(71 \%)$. También es 


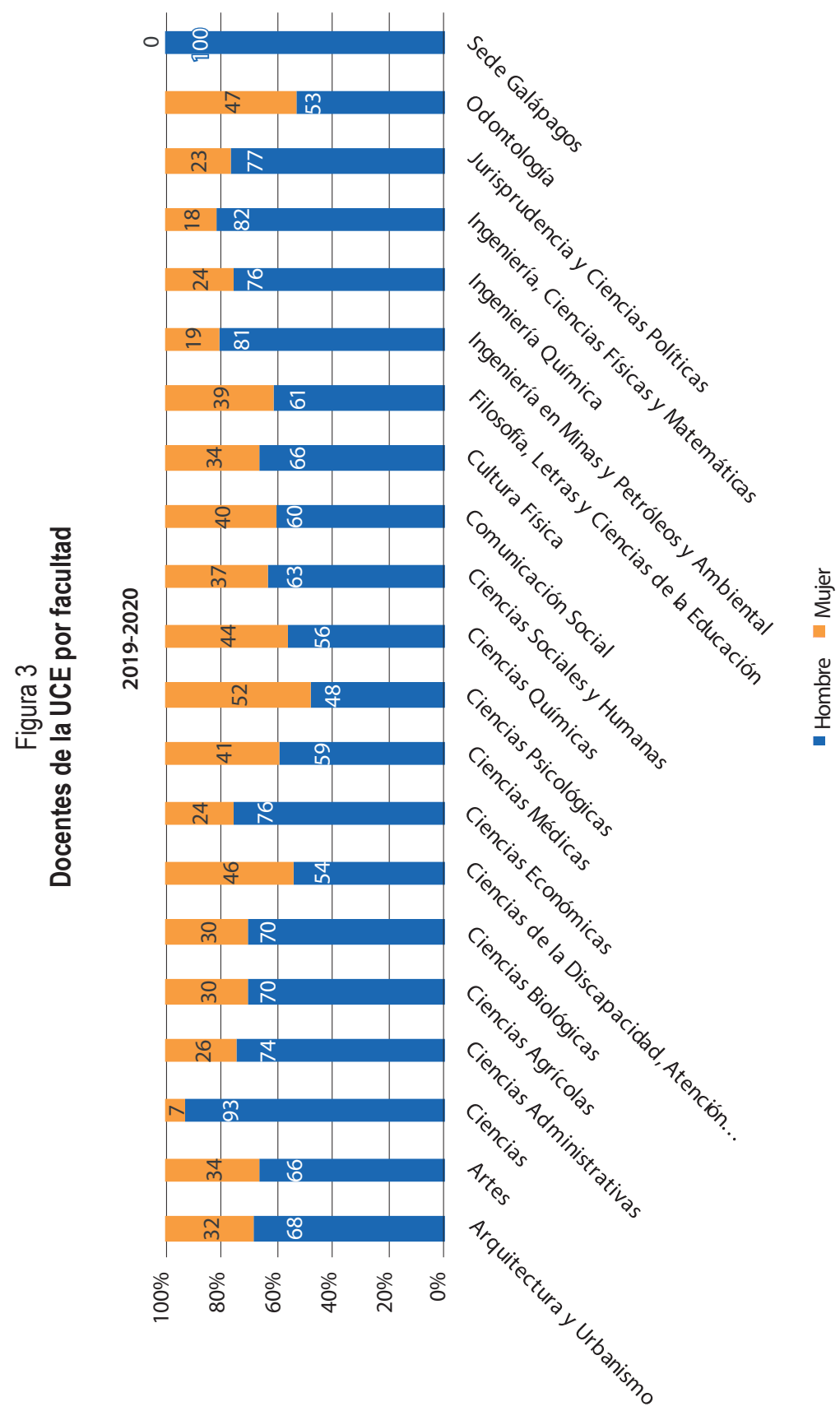

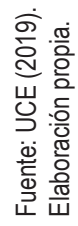


importante evidenciar la inexistencia de proyectos relacionados con género. En la tabla 1 se presenta la información respecto a la dirección de proyectos de género clasificados según sexo y facultad.

Tabla 1

\section{Proyectos de investigación de la Universidad Central del Ecuador Período 2018}

\begin{tabular}{|r|l|c|c|c|}
\hline \multirow{2}{*}{ No. Facultad } & & \multirow{2}{*}{$\begin{array}{c}\text { Proyectos } \\
\text { de género }\end{array}$} & \multicolumn{2}{c|}{ Dirección } \\
\cline { 4 - 5 } & & & Mujer & Hombre \\
\hline 1 & Ciencias Agrícolas & - & 3 & 6 \\
\hline 2 & Ciencias de la Discapacidad & - & 1 & - \\
\hline 3 & Ciencias Médicas & - & 1 & 5 \\
\hline 4 & Ciencias Psicológicas & - & 1 & 1 \\
\hline 5 & Educación Física & - & - & 1 \\
\hline 6 & Medicina Veterinaria y Zootecnia & - & 4 & 6 \\
\hline 7 & Odontología & - & 1 & - \\
\hline 8 & Ciencias Administrativas & - & 2 & 6 \\
\hline 9 & Ciencias Económicas & - & - & 6 \\
\hline 10 & Comunicación Social & - & 2 & - \\
\hline 11 & Filosofía, Letras y Ciencias de la Educación & - & 1 & 4 \\
\hline 12 & Artes & - & - & 1 \\
\hline 13 & Ciencias Sociales y Humanas & - & 3 & 3 \\
\hline 14 & Jurisprudencia & - & 1 & 1 \\
\hline 15 & Instituto Académico de Idiomas & - & - & - \\
\hline 16 & Ciencias Químicas & - & 1 & 2 \\
\hline 17 & Ingeniería Química & - & 2 & 5 \\
\hline 18 & Ingeniería en Ciencias Físicas y Matemáticas & - & - & 1 \\
\hline 19 & Ingeniería en Geología, Minas, Petróleo y Ambiental & - & - & 2 \\
\hline 20 & Arquitectura y Urbanismo & - & - & 7 \\
\hline 21 & Ciencias Biológicas & - & 2 \\
\hline & & & 1 & \\
\hline
\end{tabular}




\begin{tabular}{|r|l|c|c|c|}
\hline 22 & $\begin{array}{l}\text { FIGEMPA, revista de Investigación y Desarrollo de la } \\
\text { Facultad de Ingeniería, Minas, Petróleo y Ambiente }\end{array}$ & - & - & - \\
\hline 23 & Voluntariado Corporativo IBM & - & - & - \\
\hline 24 & ClZ, Centro Internacional de Zoonosis & - & - & - \\
\hline 25 & Prometeo & - & - & - \\
\hline & Total & 0 & 24 & 59 \\
\hline
\end{tabular}

Fuente: UCE (2019).

Elaboración propia.

En cuanto a las investigaciones realizadas en el período 2014-2018, se puede apreciar que se presenta una mayor participación femenina en la dirección: del $17 \%$ en 2014 se incrementa a $29 \%$ en 2018 . Se concluye que existe un mínimo, pero constante crecimiento en la participación de las docentes en esta área del quehacer universitario. El detalle se presenta en la figura 4.

Figura 4

\section{Proyectos de investigación: dirección por sexo}

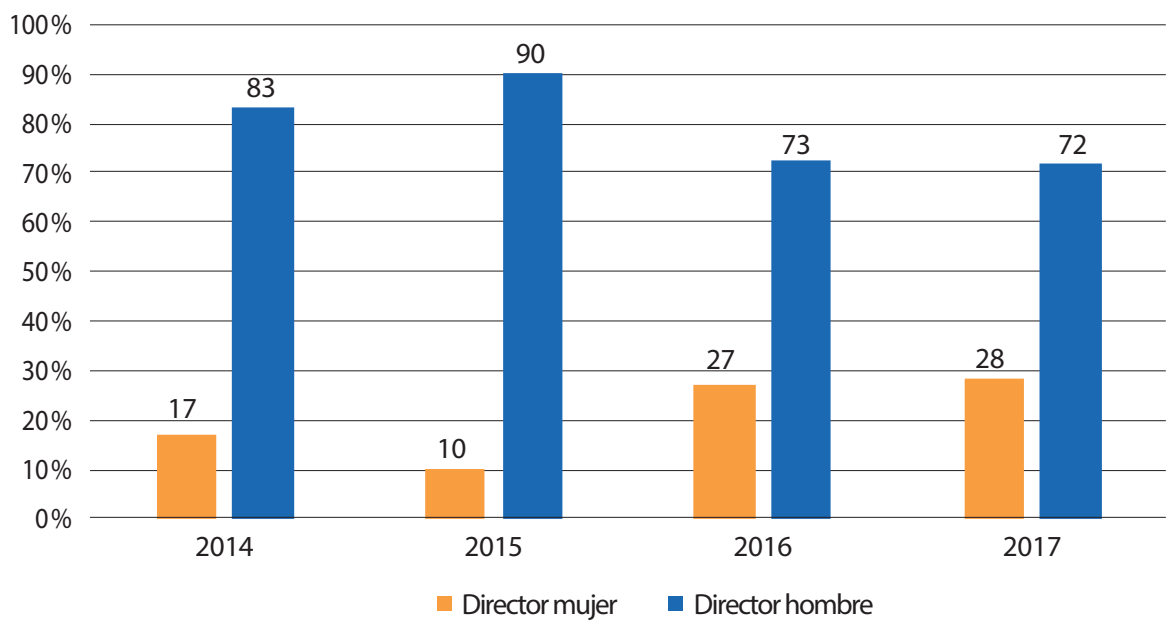

Fuente: UCE (2019).

Elaboración propia. 
En lo que tiene que ver con investigaciones relacionadas con género, en 2016 se presentó un proyecto y en 2017 dos, lo que evidencia el mínimo tratamiento de investigaciones sobre esta temática.

\section{Vinculación con la sociedad}

Según datos de la UCE (2019), el eje de vinculación con la sociedad, durante el período 2016-2018, desarrolló 168 proyectos cada semestre, con la participación de docentes y estudiantes en los territorios, para lo cual cuentan con un modelo denominado "Macroterritorios", que integra sectores rural y urbano; docencia-vinculación-investigación; apoya el aprendizaje de los estudiantes acorde con su perfil de carrera; articula en sus proyectos la competencia de los gobiernos autónomos descentralizados; conforma las comunidades de aprendizaje; $\mathrm{y}$, genera investigaciones útiles para la toma de decisiones en el territorio. La crítica a este modelo es que ha incluido temas relacionados con la equidad de género o un enfoque de género como criterio transversal.

\section{Cumplimiento ODS 5 (meta 5.5) en la UCE}

Para valorar el cumplimiento del ODS 5, su meta 5.5, "Asegurar la participación plena y efectiva de las mujeres y la igualdad de oportunidades de liderazgo a todos los niveles decisorios en la vida política, económica y pública" en la UCE, se utilizó el indicador establecido por la ONU: proporción de mujeres en cargos directivos. Los principales estamentos decisorios de la UCE son los siguientes:

- Consejo Universitario.

- Rector y vicerrectores.

- Decanatos, subdecanos y directores de carrera.

\section{Miembros del Honorable Consejo Universitario}

El Consejo Universitario, máximo órgano de Gobierno de la UCE, está conformado por el rector, vicerrector académico, vicerrector de investigaciones y vicerrector administrativo, decanos de facultades, representantes: de 
docentes de cada facultad, estudiantiles, de la Federación de Asociaciones de Profesores de la Universidad Central del Ecuador (FAPUC), de los servidores y trabajadores, de la asociación de empleados y de la Federación de Estudiantes Universitarios del Ecuador. Actualmente el Consejo Universitario está conformado por 62 integrantes.

Al analizar la conformación del Consejo Universitario, 21 son mujeres (34\%) y 41 son hombres (66\%). No se cumple el principio de paridad de género, siendo la brecha de un $16 \%$. Es importante tomar en cuenta que en esta instancia la mayoría de sus integrantes son de elección; para cambiar la correlación de fuerzas, se debe promover mayor participación femenina en los diferentes movimientos universitarios internos, además de exigir el cumplimiento de las disposiciones de la LOES (ver figura 5).

Figura 5

\section{Miembros del Honorable Consejo Universitario}

(2019)

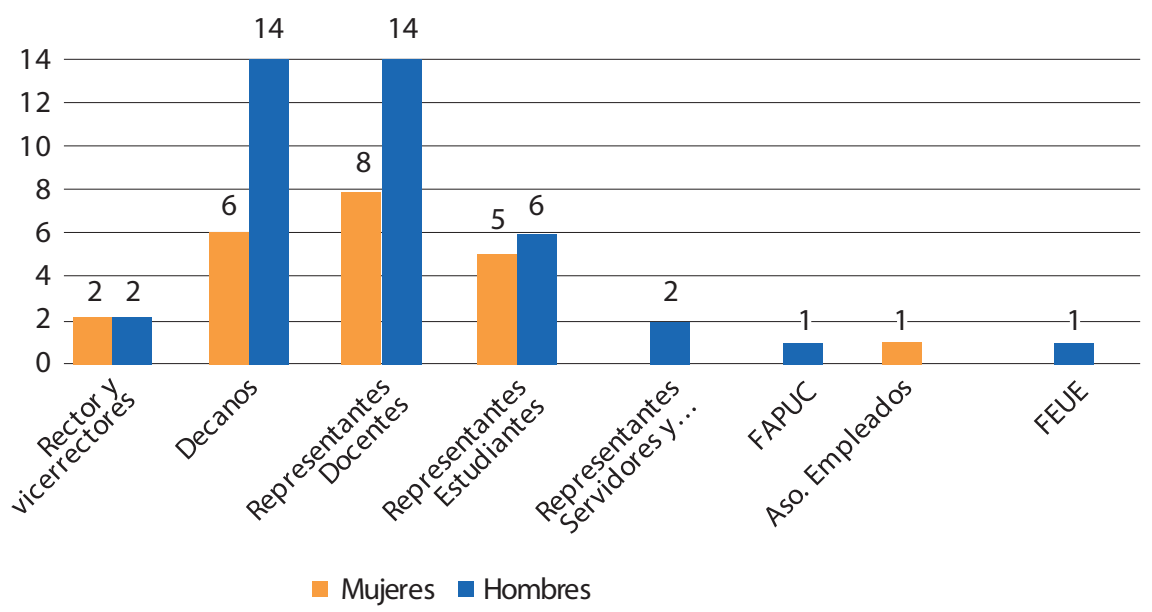

Fuente: UCE (2019).

Elaboración propia. 
Rector, vicerrectora académica, vicerrector administrativo $y$ vicerrectora de investigaciones

Las principales autoridades de la UCE son rector, vicerrector académico, vicerrector administrativo y vicerrector de investigaciones. Actualmente conformado por rector y vicerrector administrativo (hombres) y vicerrectora académica y de posgrado y vicerrectora de investigaciones, doctorados e innovación (mujeres). Se cumple con el principio de paridad. Es importante anotar que es la primera vez en la historia de la UCE que se cuenta con mujeres ocupando el puesto de Vicerrectorado; sin embargo, quien encabeza las principales autoridades continúa siendo un hombre (ver figura 6).

Figura 6

\section{Principales autoridades de la UCE}

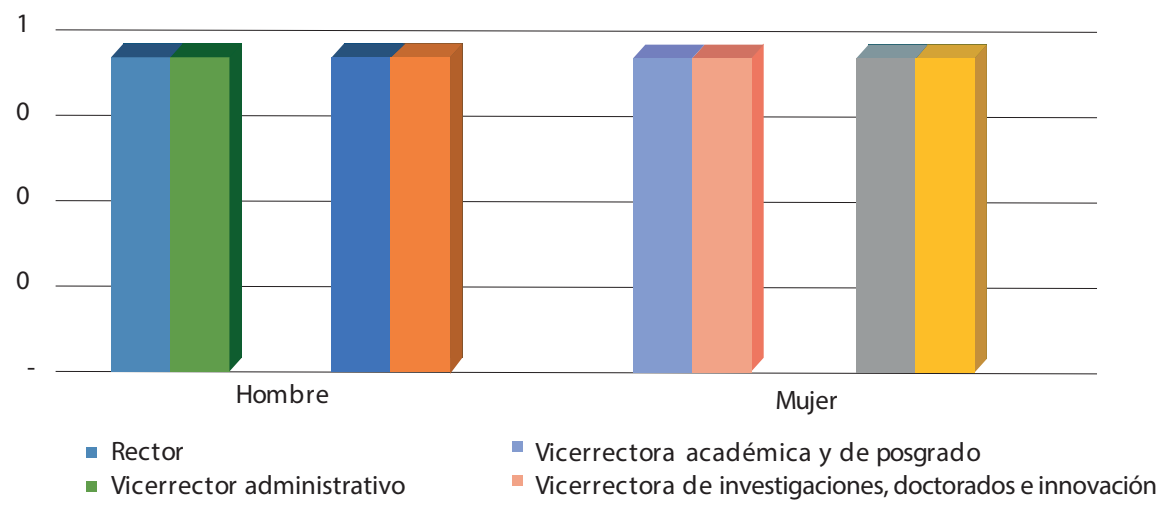

Fuente: UCE (2019).

Elaboración propia.

Decanos, subdecanos y directores de carrera

Los decanos, subdecanos y directores de carrera son designados por el rector; con un total de 20 facultades, se encontró que hay seis mujeres ocupando el puesto de decano y 14 hombres ocupando la misma posición; 6 subdecanas y 13 subdecanos (no se registra subdecano en la Facultad de 
Ciencias Químicas); 15 directoras y 36 directores de carrera. A este nivel existe una participación femenina inferior a la tercera parte de la masculina, que es proporcional a la menor participación de las mujeres en la docencia $(34 \%)$. En todo caso, queda claro que este tipo de designaciones respecto a género le corresponden al rector.

Figura 7

\section{Autoridades de las facultades de la UCE}

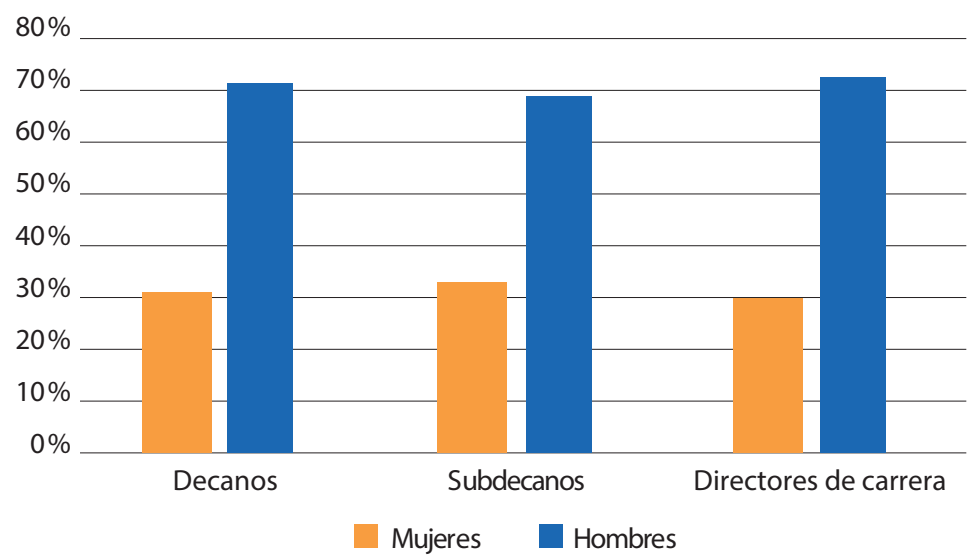

Fuente: UCE (2019).

Elaboración propia.

\section{Conclusiones}

Existen diagnósticos detallados sobre equidad de género en la universidad que tratan elementos tanto objetivos como subjetivos; sin embargo, si bien se identifican problemáticas, se establecen acuerdos, lineamientos, políticas y metas, en la práctica los avances son lentos, lo cual se explica porque el liderazgo está ejercido por hombres que no tienen como prioridad y/o no visibilizan la problemática de género en su real dimensión y, de otra parte, las mujeres tampoco se han concientizado suficientemente en las diferentes formas en las que se presenta la discriminación de género, muchas de las 
cuales son casi imperceptibles. En el caso ecuatoriano, hay que reconocer el rol fundamental que ha tenido la LOES, pues la mayoría de universidades, no por decisión propia sino por el requerimiento de la ley, han incorporado docentes mujeres en puestos de decisión, especialmente a nivel de vicerrectorados y algo menos en rectorados.

Uno de los principales cuestionamientos a la investigación fue por qué se realizó el análisis de género tomando como referente el ODS 5, puesto que se considera que la base de la equidad de género se encuentra en los propios instrumentos de derechos humanos y los ODS son el piso mínimo que debe sustentar todo Estado. La respuesta es que ningún país en el mundo cumple con el mínimo establecido en el ODS 5.

Se considera fundamental poner en práctica la transversalización de temas de equidad de género en mallas curriculares, programas de pregrado y posgrado, así como también en los procesos de investigación y vinculación con la comunidad, lo que permitiría superar los mecanismos intangibles de exclusión y superar las barreras internas de resignación y pasividad.

Es importante el uso de indicadores para conocer el estado de una meta, pero no es suficiente, pues deben ir acompañadas de análisis cualitativos para conocer las razones que generan esos resultados; por tanto, los indicadores ODS, así como los desarrollados por las autoridades de la educación superior del Ecuador, aplicables a la equidad de género, se consideran insuficientes. De igual manera, los requerimientos de la LOES para los ascensos de los docentes, como por ejemplo el número de publicaciones, investigaciones, etc., no hacen alusión a su calidad, respecto al contenido e impacto de los trabajos académicos, sino solo a la cantidad en número, lo cual desvirtúa la esencia de la educación superior.

A la pregunta de investigación formulada: ¿se está dando cumplimiento al ODS 5 y su meta 5.5 en el caso de las mujeres docentes en posición de liderazgo, respecto a puestos de elección, de designación y aportes en investigación, en la UCE?, la respuesta es no. Se han presentado avances, pero el promedio de la participación femenina en todos los órganos de dirección de la UCE es del $37 \%$. Además, se debe tomar en cuenta que la mayoría de funciones de liderazgo docente son designados por el rector (decanos, subdecanos, directores de carrera) que da como resultado que el promedio de participación femenina sea solo del $30 \%$. 
Se sugiere que, para motivar la participación femenina en procesos de investigación se podría implementar, como en el caso de Reino Unido, un sello de calidad denominado Antena Swan, que premia a las instituciones de investigación más igualitarias. Este sello les da puntos para facilitar a las universidades el acceso a financiamiento de proyectos e investigaciones.

Lamentablemente, algunas de las docentes que se encuentran desempeñando puestos de liderazgo no advierten la desigualdad, y en muchos casos, de manera consciente y la mayoría de forma inconsciente, se constituyen en entes reproductores de la cultura patriarcal. Se recomienda, por tanto, incorporar asesoría experta a las mujeres en posiciones de decisión, que les permita visibilizar, enfrentar y superar los elementos sutiles de discriminación de género en las universidades, para lograr un real empoderamiento y eficaz liderazgo femenino.

\section{Referencias}

Agencia Española de Cooperación Internacional para el Desarrollo (AECID). 2017. Estudio sobre la Cooperación de la Empresa a la Agenda de los Objetivos de Desarrollo Sostenible (ODS). Madrid: AECID.

Agencia para la Calidad de la Educación Superior de Cataluña. 2019. Marco General para la Incorporación de la Perspectiva de Género en la Docencia Universitaria. Barcelona: Agència per a la Qualitat del Sistema.

Baeza, Andrea, y Silvia Lamadrid. 2019. "¿Igualdad en la academia? Barreras de género e iniciativas en una universidad pública (2013-2018)". Pensamiento Educativo. Revista de Investigación Educacional Latinoamericana 56 (1): 1-17.10.7764/PEL.56.1.2019.9.

Benavides, Andrea, Franco Barboza, Valeria Gili, María Estévez, Victoria Galoviche, Mariana Guerra, Estela Narváez y Mirta Pastran. 2015. "Liderazgo y género en la educación superior: desigualdades entre académicas y académicos en la gestión". Memorias del Seminario Internacional Calidad de la Educación Superior y Género. Revista Ciencias Sociales 1 (40): 67-78. http://revistadigital.uce.edu.ec/index.php/CSOCIALES/article/view/1250.

Buquet, Ana. 2015. "Género y educación superior: una mirada desde América Latina". En Memorias del Seminario Internacional Calidad de la Educación Superior y Género, coordinado por AECID y FLACSO, 65-83. Quito: FLACSO.

Cárdenas, Elizabeth. 2016. "El techo de cristal en el mercado laboral femenino: caso de la Universidad de las Fuerzas Armadas -ESPE- en la ciudad de Quito en el período 20092014". Tesis de maestría, Universidad Andina Simón Bolívar, Sede Ecuador, Quito. http:// hdl.handle.net/10644/5327. 
Comisión Económica para América Latina y el Caribe (CEPAL). 2016. Horizontes 2030. La igualdad en el centro del desarrollo sostenible (LC/G. 2660. SES. 36/3). Santiago: CEPAL.

---. 2017. Estrategia de Montevideo para la Implantación de la Agenda Regional de Género en el Marco del desarrollo sostenible hacia 2030. Santiago: CEPAL.

Consejo Empresarial para el Desarrollo Sostenible (CEMDES). 2019. "Empresas y los Objetivos de Desarrollo Sostenible (ODS) en Ecuador”. ¿Cuán presentes están los ODS en los informes? Guayaquil. Accedido 20 de diciembre. https://www.cemdes.org/.

Consejo Nacional de Igualdad de Género. 2014. La violencia de género contra las mujeres en el Ecuador: Análisis de los resultados de la Encuesta Nacional sobre relaciones familiares y violencia de género contra las mujeres. Quito: El Telégrafo.

Cooper, Jennyfer. 2015. "Por qué la meritocracia traiciona a las mujeres académicas en las Instituciones de Educación Superior? Análisis y propuestas". En Memorias del Seminario Internacional Calidad de la Educación Superior y Género, coordinado por AECID y FLACSO, 131-151. Quito: FLACSO.

Díaz, Valentín. 2018. "Una mayor participación femenina en la ciencia todavía es un reto en el país". El Comercio. 26 de septiembre. https://bit.ly/3g03Rsc.

EC Defensoría del Pueblo. 2016. Política Institucional de Igualdad de Género 2016-2019. Quito: Defensoría del Pueblo.

---. 2018. Ley Orgánica de Educación Superior. Registro Oficial 53, Suplemento 298, 2 de agosto.

EC Secretaría Nacional de Educación Superior, Ciencia, Tecnología e Innovación (SENESCYT). 2015. Construyendo igualdad en la educación superior: fundamentos y lineamientos para transversalizar los ejes de igualdad y ambiente. Quito: SENESCYT / UNESCO.

EC Secretaría Técnica de Planificación y Desarrollo (SENPLADES). 2014. Agenda Nacional de las Mujeres e Igualdad de Género 2014-2017. Quito: El Telégrafo.

---. 2018. Examen Nacional Voluntario. Ecuador 2018: Objetivos de Desarrollo Sostenible. Quito: SENPLADES.

ES Ministerio de Ciencia, Innovación y Universidades. 2017. Informe Científicas en Cifras 2017. Madrid: Ministerio de Ciencia, Innovación y Universidades.

ES Ministerio de Economía y Competitividad. 2015. Plan de acción para la igualdad de oportunidades de mujeres y hombres en la sociedad de la información. 2014-2017. Madrid: Instituto de la Mujer y para la Igualdad de Oportunidades.

Fundación Española para la Ciencia y Tecnología. 2017. Científicas en cifras. Madrid. Ministerio de Innovación, Ciencia y Universidades.

Fundación FUHEM. 2019. "ODS: una revisión crítica”. En Análisis ecosocial. Madrid: https: //bit.ly/3ezrV4R.

Gómez, Carlos. 2017. "Objetivos de Desarrollo Sostenible (ODS): una revisión crítica". PAPELES de Relaciones Ecosociales y Cambio Global 140: 107-118. https://bit.ly/3cNqBd4.

Hidalgo, Antonio, Santiago García, Patricia Cubillo y Nancy Medina. 2018. Los objetivos del Buen Vivir. Una crítica de los objetivos de desarrollo sostenible y una propuesta alternativa transmoderna. Huelva: Bonanza. 
La Universidad en Internet. 2020. "Cada vez más mujeres estudian carreras universitarias para progresar en el Ecuador". UNIR. 4 de marzo. https://bit.ly/3fZFta5.

Larrea, Ana. 2015. "De la inclusión a la justicia económica: el rol de la educación superior". En Memorias del Seminario Internacional Calidad de la Educación Superior y Género, coordinado por AECID y FLACSO, 215-230. Quito: FLACSO.

Lis, Jenny. 2012. "Análisis de los grupos de investigación colombianos en ciencias económicas desde una perspectiva de género". Revista Facultad de Ciencias Económicas 20 (2): 143-164. https://bit.ly/3f53tby.

Logroño, Mercy. 2015. "Relaciones de género y poder desde las voces de las académicas: el caso de la Universidad Central del Ecuador". En Memorias del Seminario Internacional Calidad de la Educación Superior y Género, coordinado por AECID y FLACSO, 45-66. Quito: FLACSO.

Logroño, Mercy, Germania Borja y Cristina Orozco. 2018. "Visión crítica del currículo desde el enfoque de género e interseccionalidad: caso de la Universidad Central del Ecuador". Revista entorno (66): 237-255.

Manami, Ana. 2015. "Microgeopolítica del poder: la particularidad rectora de la Estatal Mayor de San Simón". En Memorias del Seminario Internacional Calidad de la Educación Superior y Género, coordinado por AECID y FLACSO, 27-44. Quito: FLACSO.

Martínez, María, y María Urrea. 2015. "Las voces de las líderes de la Universidad de Alicante sobre cuestiones de género". En Memorias del Seminario Internacional Calidad de la Educación Superior y Género, coordinado por AECID y FLACSO, 15-26. Quito: FLACSO.

Medina, Pablo, Marina Gama y Luis Núñez. 2017. "La universidad española lejos de la paridad en las cátedras". El Mundo. Accedido enero de 2020. https://bit.ly/31dJDqO.

Merma-Molina, Gladys, y María Ávalos Ramos. 2018. "El liderazgo estudiantil y el género: causas de su elección y problemáticas en su desempeño". Revista Ciencias Sociales, 1 (40): 143-156. http://revistadigital.uce.edu.ec/index.php/CSOCIALES/article/view/1256.

Mora, Enrico, y Margot Pujal. 2009. Introducción a la perspectiva de género en la docencia universitaria. Girona: Publicación INIVEST 09. https://bit.ly/3fSzUKA.

ONU Mujeres. 2015. La Asamblea General adopta la Agenda 2030 para el Desarrollo Sostenible. Nueva York: Naciones Unidas. https://bit.ly/2NuVU1Z.

--- 2018. "Hacer las promesas realidad: la igualdad de género en la Agenda 2030 para el desarrollo sostenible". ONU Mujeres. Nueva York. https://bit.ly/2VcsB8W.

---. 2019. Objetivo 5: Lograr la igualdad entre los géneros y empoderar a todas las mujeres y niñas. Lima: Naciones Unidas. https://onu.org.pe/ods-5/.

Universidad Central del Ecuador. 2019. "Servicios". Universidad Central del Ecuador. Accedido 12 de diciembre. https://www.uce.edu.ec/web/trasparencia.

Wappenetein, Susana. 2016. “¿Quién toma las decisiones? Relaciones de género y puestos de toma de decisión en la Universidad Central del Ecuador". Tesis de maestría en Ciencias Sociales con mención en Género y Desarrollo, FLACSO, Quito. https://bit.ly/2Z1kZqF. 